\title{
LETTER
}

Multiple myeloma gammopathies

\section{Clinical features and survival outcomes in IgD myeloma: a study by Asia Myeloma Network (AMN)}

\author{
Jin Liu ${ }^{1}$ Xiaoxia Hu $\mathbb{1}^{2} \cdot$ Yanchun Jia ${ }^{1} \cdot$ Jin $\mathrm{Lu}^{3} \cdot$ Jae Hoon Lee ${ }^{4} \cdot$ Kihyun Kim $\mathbb{1}^{5} \cdot$ Wenming Chen ${ }^{6} \cdot$ Aijun Liu $^{6}$. \\ Yang Liu ${ }^{3} \cdot$ Qi Chen ${ }^{7}$. Chunyang Zhang $\mathbb{1}^{1,8} \cdot$ Cheolwon Suh $\rrbracket^{9} \cdot$ Min Kyoung Kim ${ }^{10} \cdot$ Fan Zhou $^{11}$. \\ Wee Joo Chng ${ }^{12,13,14} \cdot$ Shaji K. Kumar $\mathbb{( D )}^{15} \cdot$ Brian Durie $^{16} \cdot$ Jian Hou $\mathbb{( i D}^{17} \cdot$ Weijun Fu $\left(\mathbb{D}^{1} \cdot\right.$ Juan Du (i)
}

Received: 3 July 2020 / Revised: 21 August 2020 / Accepted: 5 October 2020 / Published online: 20 October 2020

(c) The Author(s) 2020. This article is published with open access

\section{To the Editor:}

Immunoglobulin D (IgD) myeloma is a rare isotype that comprises 1-2\% of multiple myeloma (MM) patients [1-3], which has significantly inferior survival for a median overall survival (OS) between 13 and 21 months [4-6].

These authors contributed equally: Jin Liu, Xiaoxia Hu, Yanchun Jia

These authors contributed equally: Jian Hou, Weijun Fu, Juan Du

Supplementary information The online version of this article (https:// doi.org/10.1038/s41375-020-01060-w) contains supplementary material, which is available to authorized users.

\footnotetext{
Jian Hou

houjian@medmail.com.cn

$\bowtie$ Weijun Fu

fuweijun2010@ hotmail.com

$\triangle$ Juan Du

juan_du@live.com
}

1 Department of Hematology, Myeloma \& Lymphoma Center, Shanghai Changzheng Hospital, The Second Military Medical University, Shanghai, China

2 State Key Laboratory of Medical Genomics, Shanghai Institute of Hematology, National Research Center for Translational Medicine, Shanghai Rui Jin Hospital, Shanghai Jiao Tong University School of Medicine, Shanghai, China

3 Peking University Institute of Hematology, Peking University People's Hospital, Beijing, China

4 Incheon Regional Cancer Center, Gil Medical Center, Gachon University College of Medicine, Incheon, Republic of Korea

5 Department of Medicine, Samsung Medical Center, Sungkyunkwan University School of Medicine, Seoul, Republic of Korea

6 Department of Hematology, Beijing Chaoyang Hospital, Capital Medical University, Beijing, China
Given the lack of large cohort with comprehensive clinical and cytogenetic assessment, knowledge about IgD myeloma is obtained mostly from a limited sample size [7]. Therefore, we carried out a multicenter retrospective study to evaluate the prevalence, clinical features, prognosis, and to develop and validate a prognostic model, including 356 patients with IgD myeloma from 14 centers of Asian Myeloma Network (AMN).

Data were collected from China, Korea, and Singapore diagnosed from 2002 to 2019 (Supplementary Table 1). Ethical committee approvals were obtained and study protocol was approved by the Institutional Review Board of each institution. To avoid clinical information leak, and get

7 Department of Health Statistics, Second Military Medical University, Shanghai, China

8 School of Medicine, University of Maryland Baltimore, Baltimore, Maryland, USA

9 Department of Oncology, Asan Medical Center, University of Ulsan College of Medicine, Seoul, Republic of Korea

10 Department of Internal Medicine, Yeungnam University Medical Center, Yeungnam University College of Medicine, Daegu, Republic of Korea

11 Department of Hematology \& Oncology, Jingan District Zhabei Central Hospital Shanghai, Shanghai, China

12 Department of Hematology-Oncology, National University Cancer Institute of Singapore, National University Health System, Singapore, Singapore

13 Cancer Science Institute of Singapore, Singapore, Singapore

14 Department of Medicine, Yong Loo Lin School of Medicine, National University of Singapore, Singapore, Singapore

15 Division of Hematology, Department of Internal Medicine, Mayo Clinic, Rochester, MN, USA

16 Cedars-Sinai Medical Center, Los Angeles, CA, USA

17 Department of Hematology, Renji Hospital, Shanghai Jiao Tong University School of Medicine, Shanghai, China 
Table 1 Characteristics of the study populations.

\begin{tabular}{|c|c|c|c|c|c|c|c|}
\hline Variable & $\begin{array}{l}\text { IgD MM } \\
(N=356, \%)\end{array}$ & $\begin{array}{l}\text { Non-IgD MM } \\
(N=712, \%)\end{array}$ & $P$ value & Variable & $\begin{array}{l}\text { IgD MM } \\
(N=356, \%)\end{array}$ & $\begin{array}{l}\text { Non-IgD MM } \\
(N=712, \%)\end{array}$ & $P$ value \\
\hline Sex & & & & $\leq 0.01, \geq 100$ & $138(38.8)$ & $356(50)$ & \\
\hline Male & $241(67.7)$ & $429(60.3)$ & 0.018 & Data missing & $81(22.7)$ & $0(0)$ & \\
\hline Female & $115(32.3)$ & $283(39.7)$ & & $\operatorname{Del}(13 q)$ in FISH & & & \\
\hline \multicolumn{2}{|c|}{ Age at diagnosis,years } & & & Yes & $77(21.7)$ & $261(36.7)$ & 0.001 \\
\hline Median (range) & $56(32-85)$ & $62(23-96)$ & $<0.001$ & No & $224(62.9)$ & $451(63.3)$ & \\
\hline$<65$ & $286(80.3)$ & $468(65.7)$ & & Data missing & $55(15.4)$ & $0(0)$ & \\
\hline$\geq 65$ & 70 (19.7) & $244(34.3)$ & & $\operatorname{Del}(17 p)$ in FISH & & & \\
\hline \multicolumn{2}{|l|}{ DS Stage } & & & Yes & $35(9.9)$ & $75(10.5)$ & 0.609 \\
\hline I & $8(2.2)$ & $16(2.2)$ & 0.812 & No & $266(74.7)$ & $637(89.5)$ & \\
\hline II & $23(6.5)$ & $54(7.6)$ & & Data missing & $55(15.4)$ & $0(0)$ & \\
\hline III & $323(91.3)$ & $642(90.2)$ & & 1q21 gains in FISH & & & \\
\hline \multicolumn{2}{|l|}{ ISS stage } & & & Yes & $91(25.6)$ & $368(51.7)$ & $<0.001$ \\
\hline I & $70(19.7)$ & $179(25.1)$ & $<0.001$ & No & $179(50.2)$ & $344(48.3)$ & \\
\hline II & $64(17.9)$ & $262(36.8)$ & & Data missing & $86(24.2)$ & $0(0)$ & \\
\hline III & $222(62.4)$ & $271(38.1)$ & & $\mathrm{t}(11 ; 14)$ in FISH & & & \\
\hline \multicolumn{2}{|c|}{ Plasma cells of BM (\%) } & & & Yes & $88(24.7)$ & $96(13.5)$ & $<0.001$ \\
\hline$\geq 50$ & 149 (41.9) & $143(20.1)$ & $<0.001$ & No & $213(59.9)$ & $616(86.5)$ & \\
\hline$<50$ & $207(58.1)$ & $569(79.9)$ & & Data missing & $55(15.4)$ & $0(0)$ & \\
\hline \multicolumn{2}{|c|}{ Hemoglobin level (g/L) } & & & $\mathrm{t}(4 ; 14)$ in FISH & & & \\
\hline$<100$ & 231 (64.9) & $420(59)$ & 0.063 & Yes & $4(1.1)$ & $136(19.1)$ & $<0.001$ \\
\hline$\geq 100$ & $125(35.1)$ & $292(41)$ & & No & $297(83.5)$ & $576(80.9)$ & \\
\hline \multicolumn{2}{|c|}{ Platelet count $\left(10^{9} / \mathrm{L}\right)$} & & & Data missing & $55(15.4)$ & $0(0)$ & \\
\hline$<100$ & $76(22.3)$ & $91(12.8)$ & $<0.001$ & $\mathrm{t}(14 ; 16)$ in FISH & & & \\
\hline$\geq 100$ & $280(78.7)$ & $621(87.2)$ & & Yes & $3(0.9)$ & $8(1.1)$ & 0.859 \\
\hline \multicolumn{2}{|l|}{ Serum LDH (U/L) } & & & No & $298(83.7)$ & $704(98.9)$ & \\
\hline$\geq 245$ & $136(38.2)$ & $177(24.9)$ & $<0.001$ & Data missing & $55(15.4)$ & $0(0)$ & \\
\hline$<245$ & $220(61.8)$ & $535(75.1)$ & & Double hit ${ }^{\mathrm{a}}$ & & & \\
\hline \multicolumn{2}{|c|}{ Serum creatinine level (mg/dL) } & & & yes & $20(5.6)$ & $116(16.3)$ & $<0.001$ \\
\hline$\geq 2$ & $137(38.5)$ & $131(18.4)$ & $<0.001$ & no & $251(70.5)$ & $596(83.7)$ & \\
\hline$<2$ & $219(61.5)$ & $581(81.6)$ & & Data missing & $85(23.9)$ & $0(0)$ & \\
\hline \multicolumn{2}{|c|}{ Serum calcium level (mmol/L) } & & & Triple hit ${ }^{b}$ & & & \\
\hline$\geq 2.65$ & $85(23.9)$ & $105(14.7)$ & $<0.001$ & Yes & $1(0.3)$ & $11(1.5)$ & 0.135 \\
\hline$<2.65$ & $271(76.1)$ & $607(85.3)$ & & No & $269(75.6)$ & $701(98.5)$ & \\
\hline \multicolumn{2}{|c|}{ Light chain restriction } & & & Data missing & $86(24.1)$ & $0(0)$ & \\
\hline Kappa & $40(11.2)$ & $407(57.2)$ & $<0.001$ & $\mathrm{t}(11 ; 14)$ and Del ( & ) in FISH & & \\
\hline Lambda & $316(88.8)$ & $305(42.8)$ & & Yes & $28(7.9)$ & $28(3.9)$ & 0.001 \\
\hline \multicolumn{2}{|c|}{ Extramedullary plasmacytoma } & & & No & $273(76.7)$ & $684(96.1)$ & \\
\hline Yes & $68(19.1)$ & $106(14.9)$ & 0.079 & Data missing & $55(15.4)$ & $0(0)$ & \\
\hline No & $288(80.9)$ & $606(85.1)$ & & $\mathrm{t}(11 ; 14)$ and Del ( & ) in FISH & & \\
\hline \multicolumn{2}{|l|}{ R-ISS stage } & & & Yes & $10(2.8)$ & $5(0.7)$ & 0.002 \\
\hline I & $42(11.8)$ & $114(16)$ & $<0.001$ & No & $291(81.8)$ & 707 (99.3) & \\
\hline II & $175(49.2)$ & $449(63.1)$ & & Data missing & $55(15.4)$ & $0(0)$ & \\
\hline III & $115(32.3)$ & $149(20.9)$ & & $\mathrm{t}(11 ; 14)$ and $1 \mathrm{q} 21$ & ins in FISH & & \\
\hline Data missing & $24(6.7)$ & $0(0)$ & & Yes & $27(7.6)$ & $39(5.5)$ & 0.012 \\
\hline \multicolumn{2}{|l|}{ FLCR } & & & No & $243(68.2)$ & $673(94.5)$ & \\
\hline 0.01-100 & $137(38.5)$ & $356(50)$ & 0.959 & Data missing & $86(24.2)$ & $0(0)$ & \\
\hline
\end{tabular}

Table 1 (continued)

609

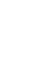


Table 1 (continued)

\begin{tabular}{lccr}
\hline Variable & $\begin{array}{c}\text { IgD MM } \\
(N=356, \%)\end{array}$ & $\begin{array}{c}\text { Non-IgD MM } \\
(N=712, \%)\end{array}$ & $P$ value \\
\hline $\mathrm{t}(11 ; 14)$ and t $(4 ; 14)$ in FISH & & \\
Yes & $1(0.3)$ & $0(0)$ & 0.124 \\
No & $300(84.3)$ & $712(100)$ & \\
Data missing & $55(15.4)$ & $0(0)$ & NA \\
$\mathrm{t}(11 ; 14)$ and t $(14 ; 16)$ in FISH & & \\
Yes & $0(0)$ & $0(0)$ & \\
No & $301(84.6)$ & $712(100)$ & \\
Data missing & $55(15.4)$ & $0(0)$ & \\
$\mathrm{t}(11 ; 14)$ and double hit in FISH & & \\
Yes & $6(1.7)$ & $2(0.3)$ & \\
No & $265(74.4)$ & $710(99.7)$ & \\
Data missing & $85(23.9)$ & $0(0)$ & \\
$\mathrm{t}(11 ; 14)$ and triple hit in FISH & & \\
Yes & $0(0)$ & $0(0)$ & \\
No & $270(75.8)$ & $712(100)$ & \\
Data missing & $86(24.2)$ & $0(0)$ & \\
\hline
\end{tabular}

$M M$ multiple myeloma, Ig immunoglobulin, DS Durie Salmon, ISS international staging system, $R$-ISS revised ISS, $L D H$ Lactate dehydrogenase, $B M$ bone marrow, FISH fluorescence in situ hybridization, Del deletion, FLCR free light chains ratio, $N A$ not avaliable.

${ }^{a}$ The cooccurrence of any 2 of the following: $t(4 ; 14), t(14 ; 16)$, gain (1q), del(17p)

${ }^{b}$ The cooccurrence of 3 or more of the following: $t(4 ; 14), t(14 ; 16)$, $\operatorname{gain}(1 q), \operatorname{del}(17 p)$.

a real sense of the accurate model's outcomes, we split existing $356 \operatorname{IgD} \mathrm{MM}$ to three parts, namely training cohort (one center from Shanghai, $n=212$ ), validation cohort 1 (two centers from Beijing, $n=81$ ), and validation cohort 2 (centers from Korea and Singapore, $n=63$ ). The Least Absolute Shrinkage and Selector Operation (LASSO) Cox regression model to determine prognostic factors from the variables with $P<0.05$ in the log-rank tests was performed as described $[8,9]$. The quality of the prediction model was measured using the concordance index (C-index) and areas under the time-dependent receiver-operating characteristics (ROC) curves (AUCs). A bootstrap with 1000 re-samples was used for internal validation. SAS 9.4 and R 3.5.1 were used for the statistical analysis.

A total of 356 patients with $\operatorname{IgD}$ myeloma represented $2-8.8 \%$ of all myeloma patients, especially over $5 \% \operatorname{IgD}$ myeloma prevalence in Chinese centers. We compared the clinical characteristics of IgD myeloma with 712 (1:2) nonIgD myeloma patients random selected as control matched for year of diagnosis and systemic therapy from Shanghai Changzheng Hospital. Baseline characteristics of total cohort are listed on Table 1 and different centers are shown in Supplementary Table 2. IgD myeloma patients had a higher frequency in male, younger than 65 years, advanced
R-ISS stage III, hypercalcemia, elevated creatinine levels, and elevated LDH. Cytogenetic information was available for 301 patients $(84.6 \%$ ), while the 1q21 probe was only performed in $75.8 \%$ patients. Notably, $29.2 \%$ frequency of $\mathrm{t}(11 ; 14)$ was predominantly higher compared to those in non-IgD subtypes $(P<0.001)$. Among the $88 \operatorname{IgD}$ patients harbored $\mathrm{t}(11 ; 14)$, the most frequent chromosome abnormalities (CA) coupled with $\mathrm{t}(11 ; 14)$ were $13 \mathrm{q}$ $(31.8 \%), 1 \mathrm{q} 21+(30.7 \%)$, and followed $17 \mathrm{p}-(11.4 \%)$. 'Double-hit' or 'triple-hit' $[10,11]$ only occurred $5.6 \%$ and $0.3 \%$ patients, respectively.

And then, we compared IgD myeloma patients with IgG, $\operatorname{IgA}$, and light chain patients random selected as matched control (Supplementary Table 3). The median age was younger in IgD compared with others myeloma subtypes. Notably, the frequency of $t(11 ; 14)$ was significantly higher than non-IgD subtypes (IgD $29.2 \%$ vs $\operatorname{IgG} 10.6 \%$ vs $\operatorname{IgA}$ $8.4 \%, P<0.001$ ), but was a slight higher than light chain subtype (29.2 vs $24.9 \%$ ). 'Double-hit' phenotype was significant lower in IgD myelomas than others subtypes.

Frontline treatment modalities used are shown in Supplementary Table 4. The overall response rate (ORR) was $88.8 \%$, and very good partial response or better was $58.6 \%$ (Supplementary Table 5). After a median follow-up of 8.2, 7.3 , and 4.9 years for the three cohorts, the median OS were 36.5 months for the total cohort and 31.2 months in training cohort, 52.2 months in validation cohort 1 , and 45.7 months in validation cohort 2 (Supplementary Fig. 1 and Supplementary Table 6). Patients received IMiDs showed a relatively longer median OS than others regimens, however, which untranslated into a significant survival benefit $(P=$ 0.17, Supplementary Fig. 2a), and might be the subgroups limitation. Patients received ASCT had a median OS of 45.7 months, which was a slightly longer than 35 months for non-ASCT patients $(P=0.4$, Supplementary Fig. $2 b)$. We subsequently investigated whether cytogenetic aberration was a prognostic factor [12], which showed that CA did not have an impact on OS, suggesting other molecular events overcome initial CA risk features and impact prognosis.

Subsequently, the LASSO Cox regression model to determine prognostic factors from the univariate analysis was performed (Supplementary Table 7). Five clinical parameters with statistically relevant, including lambda light chain, plasma cells in $\mathrm{BM} \geq 50 \%$, hemoglobin $<100 \mathrm{~g} / \mathrm{L}, \mathrm{LDH} \geq$ $245 \mathrm{U} / \mathrm{L}$, and extramedullary plasmacytoma, were integrated into multivariate LASSO regression model (Supplementary Table 8). A nomogram was developed and the risk score was computed as follows: $0.9215 \times$ lambda light chain + $0.6376 \times$ plasma cells in $\mathrm{BM}(\geq 50 \%)+0.5203 \times$ anemia $(<100 \mathrm{~g} / \mathrm{L})+0.6864 \times \mathrm{LDH} \quad(\geq 245 \mathrm{U} / \mathrm{L})+0.4484 \times$ extramedullary plasmacytoma (variable present $=1$, absent $=0$, Fig. 1a, b). The predictive accuracy for OS calculated using the C-index was 0.705 (95\% CI, 0.663-0.747). In the internal 

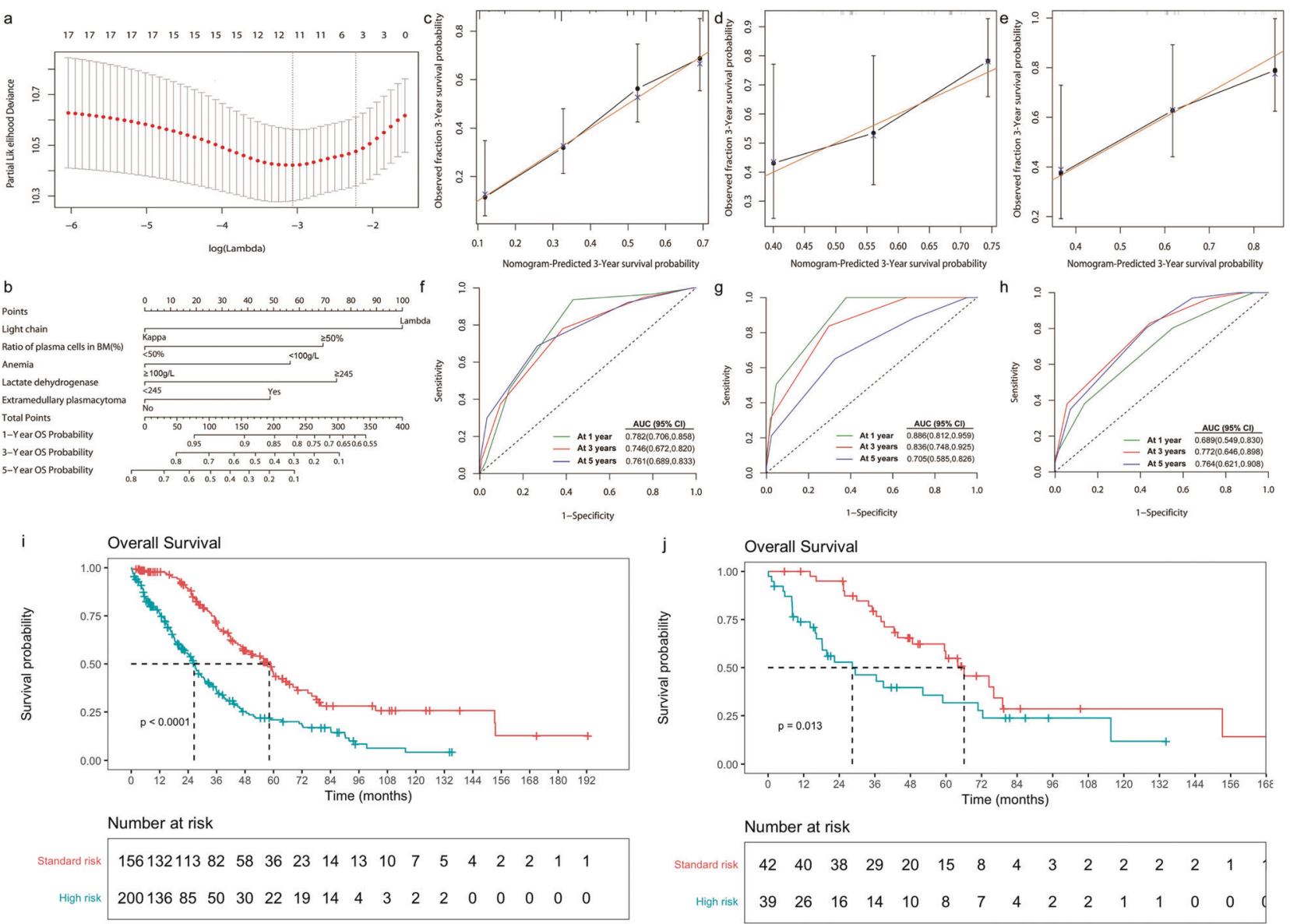

Group + Standard risk + High risk
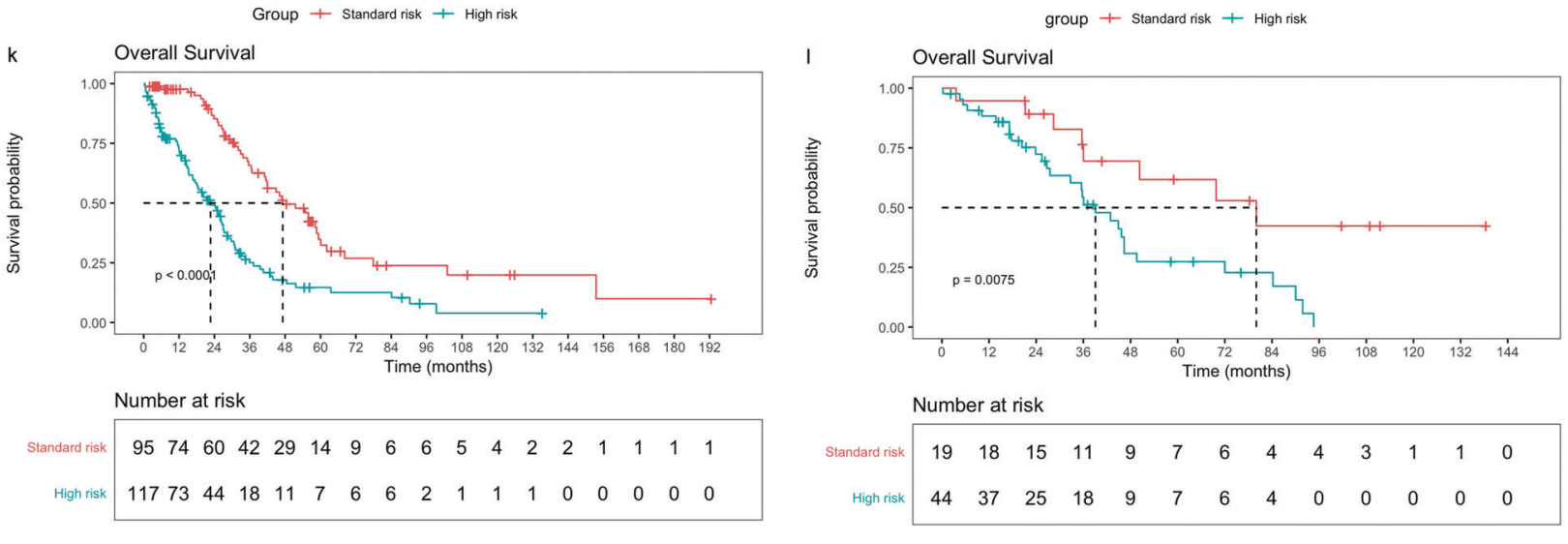

group + Standard risk + High risk

validation, the corrected C-index of OS was 0.696. Similarly, the C-index for OS in validation cohort 1 was 0.690 (95\% CI, $0.612-0.768$ ) and 0.703 (95\% CI, 0.608-0.798) in validation cohort 2 . The calibration curves of the alternative nomogram to predict the 3-year OS presented in Fig. 1c-e suggested a good fit for the observed nomogram, when compared with the ideal nomogram. The panel displayed an AUC value at 1-year, 3-year, and 5-year OS, and the validation sets had a similar high AUC values at these timepoints (Fig. 1f-h).
On the basis of the distribution of the risk scores and the 3year survival probability, two categories of risk were created with the cut-off point at 1.56: standard risk (risk score $\leq 1.56$, $n=156$ ) and high-risk subgroup (risk score $>1.56, n=200$ ). The clinical characteristics between derivations were presented in Supplementary Table 6. Patients with IgD myeloma at standard risk were significantly better than high-risk subgroup (Fig. 1i). Similar results were obtained in training and validation cohorts respectively (Fig. $1 \mathrm{j}-1)$. Notably we 
Fig. 1 Development of a predictive model and validation. LASSO Cox regression model to determine prognostic factors from the variables with $P<0.05$ in the log-rank tests was performed. The prediction model was established on the basis of variables selected from the LASSO Cox regression model and weighted using the Cox regression coefficient. Cross-validation for tuning parameter selection in the LASSO model (a). The nomogram based on data from training patients to predict individual prognosis (b). The calibration curves of an alternative nomogram to predict 3-year OS of $\operatorname{IgD}$ myeloma in training patients $(\mathbf{c})$, validation cohort 1 (d) and validation cohort $2(\mathbf{e})$. The $X$-axis represents the predicted survival probability calculated using the nomogram, while the $Y$-axis represents the actual survival probability for patients. The gold 45-degree line represents the ideal nomogram, while the black line represents the observed nomogram. The AUC values were $0.746-0.782$ (f), $0.705-0.886$ (g), and $0.689-0.772(\mathbf{h})$ in the training cohort, validation cohort 1 and validation cohort 2, respectively. On the basis of the distribution of the risk scores and the 3-year survival probability, two categories of risk were created with the cut-off point at 1.56: standard risk (risk score $\leq 1.56$, $n=156$ ) and high-risk subgroup (risk score $>1.56, n=200$ ). The 3year OS in patients with IgD myeloma at standard risk were significantly better than those of patients at high-risk subgroup (70.6 \pm $4.1 \%$ vs $36.1 \pm 3.8 \%, P<0.0001,1 i)$. Similar results were obtained in the training cohort $(65.8 \pm 5.7 \%$ vs $25.1 \pm 4.6 \%, P<0.0001,1 \mathrm{k})$, validation cohort $1(79.4 \pm 6.5 \%$ vs $46.2 \pm 8.6 \%, P=0.013,1 \mathrm{j})$, and validation cohort $2(69.5 \pm 11.5 \%$ vs $54.4 \pm 8.3 \%, P=0.0075,11)$.

identified that ASCT showed a survival advantage in highrisk group, while it did not improve the survival in standard risk group (Supplementary Fig. 3). Moreover the prediction value of the model was independent of induction modalities (Supplementary Fig. 4).

The clinical features of $\operatorname{IgD}$ myeloma in this study indicated a potentially high-risk population. We identified a higher prevalence difference may be related to the difference in ethnicity, an efficient algorithm diagnosis, and assembled patients. We found $40.6 \%$ of patients had abnormal karyotypes. Translocation $(11 ; 14)$ was predominantly high frequency, which showed the similar to others studies $[13,14]$. By far, the most clinically relevant feature of myeloma with $t$ $(11 ; 14)$ is increased expression of the anti-apoptotic protein BCL-2 [15], and venetoclax of Bcl-2 inhibitor provides a new option for $\mathrm{t}(11 ; 14)$, which may have a special significances for IgD patients with $\mathrm{t}(11 ; 14)$.

The median OS with 36.5 months in IgD myeloma showed an inferior survival than patients with more common myeloma subtypes [16, 17]. Although the ORR $(88.8 \%)$ has not improved rapidly in this AMN study, patients received IMiD with lenalidomide showed a better trend median OS than patients received PIs, conventional chemotherapy regimens, even PI + thalidomide as PI + IMiD subgroup, while PI + IMiD overcame improvement due to high peripheral neuropathy to limit the application. However, the patients received IMiD is too small to draw the definite conclusion, which might provide a clue to optimize treatment. Meanwhile, we demonstrated ASCT would benefit for IgD myeloma patients with high-risk score.
Previous studies concentrated on the prognostic value of single variable $[1,3,5,18,19]$, as it is a challenge to construct a comprehensive prognostic model due to the rarity of $\mathrm{IgD}$ myeloma. We used $356 \mathrm{IgD}$ myeloma patients to develop a prognostic model based on the 17 variables of the multivariate LASSO model to estimate the 3-year OS. Five clinical parameters were identified as clinically relevant to compute the risk score, which a high-risk score was consistently associated with a poor survival outcome. Moreover, the prediction value of the model was independent of induction modalities. This risk model improves the classification of $\operatorname{IgD}$ myeloma and may facilitate the development of risk-adapted treatment strategies. Although this retrospective design, presence of missing data, and heterogeneous treatment could have resulted in the introduction of selection bias and underestimate or overestimate of some of results, we believe that the large sample size and the multicenter study performed in such a rare disease could balance out some of these weaknesses.

In conclusion, we described the clinical features of 356 IgD myeloma patients, as well as developed and validated a predictive model containing five baseline clinical variables that could group the IgD patients into standard risk and high risk. Meanwhile, we demonstrated that IMiDs therapy might be a trend to benefit for the patient's outcome, and ASCT could benefit patient with high risk within the predictive model. These findings may provide guidance for management of IgD myeloma and better prognostic stratification for development of risk-adapted treatment strategies.

Acknowledgements We are grateful to all investigators for recruiting patients to AMN. We acknowledge the work of Aijie Huang (Institute of Hematology, Changhai Hospital, Shanghai, China) and Hao Yu (Jiangsu Key Laboratory of Zoonosis/Jiangsu Co-Innovation Center for Prevention and Control of Important Animal Infectious Diseases and Zoonose, Yangzhou University, Yangzhou, China) in data analyses. Finally, we are grateful to all patients whose participation made this study possible. This work was supported in part by National Natural Science Foundation of China (NFSC 81372543, NFSC 81870164), and Scholarship from Shanghai Health Bureau (2017BR012).

Author contributions JL, XH and YJ collected and analyzed the data, and wrote the first draft, and approved the final version of the paper; $\mathrm{JL}, \mathrm{CS}, \mathrm{MKK}, \mathrm{FZ}$, and WC performed patient management and approved the final version of the paper; YJ, AL, LY and ZY, performed patients' follow-up, participated in final data analysis and approval of the final version of the paper; QC analyzed the data and approval of the final version of the paper; JHL, KK, and WJC, performed patient management revised the paper critically, and approved the final version of the paper; SKK and BD revised the paper critically, and approved the final version of the paper; WF, and JH designed the study, performed patient management, and approved the final version of the paper, and; JD designed the study, performed patient management, analyzed the data, wrote the first draft, approved the final version of the paper. 


\section{Compliance with ethical standards}

Conflict of interest The authors declare that they have no conflict of interest.

Publisher's note Springer Nature remains neutral with regard to jurisdictional claims in published maps and institutional affiliations.

Open Access This article is licensed under a Creative Commons Attribution 4.0 International License, which permits use, sharing, adaptation, distribution and reproduction in any medium or format, as long as you give appropriate credit to the original author(s) and the source, provide a link to the Creative Commons license, and indicate if changes were made. The images or other third party material in this article are included in the article's Creative Commons license, unless indicated otherwise in a credit line to the material. If material is not included in the article's Creative Commons license and your intended use is not permitted by statutory regulation or exceeds the permitted use, you will need to obtain permission directly from the copyright holder. To view a copy of this license, visit http://creativecommons. org/licenses/by/4.0/.

\section{References}

1. Jancelewicz Z, Takatsuki K, Sugai S, Pruzanski W. IgD multiple myeloma. Review of 133 cases. Arch Intern Med. 1975;135:87-93.

2. Morris C, Drake M, Apperley J, Iacobelli S, van Biezen A, Bjorkstrand B, et al. Efficacy and outcome of autologous transplantation in rare myelomas. Haematologica. 2010;95:2126-33.

3. Blade J, Lust JA, Kyle RA. Immunoglobulin D multiple myeloma: presenting features, response to therapy, and survival in a series of 53 cases. J Clin Oncol. 1994;12:2398-404.

4. Zagouri F, Kastritis E, Symeonidis AS, Giannakoulas N, Katodritou E, Delimpasi S, et al. Immunoglobulin D myeloma: clinical features and outcome in the era of novel agents. Eur $\mathrm{J}$ Haematol. 2014;92:308-12.

5. Wechalekar A, Amato D, Chen C, Keith Stewart A, Reece D. IgD multiple myeloma-a clinical profile and outcome with chemotherapy and autologous stem cell transplantation. Ann Hematol. 2005; $84: 115-7$

6. Kuliszkiewicz-Janus M, Zimny A, Sokolska V, Sasiadek M, Kuliczkowski K. Immunoglobulin D myeloma-problems with diagnosing and staging (own experience and literature review). Leuk Lymphoma. 2005;46:1029-37.
7. Selene II, Jose JA, Khalil MJ, Faisal MS, Malik MN. Presentation patterns, diagnostic markers, management strategies, and outcomes of $\mathrm{IgD}$ multiple myeloma: a systematic review of literature. Cureus. 2019;11:e4011.

8. Ternes N, Rotolo F, Michiels S. Empirical extensions of the lasso penalty to reduce the false discovery rate in high-dimensional Cox regression models. Stat Med. 2016;35:2561-73.

9. Liu Y, Huang A, Chen Q, Chen X, Fei Y, Zhao X, et al. A distinct glycerophospholipid metabolism signature of acute graft versus host disease with predictive value. JCI Insight. 2019;5:e129494.

10. Boyd KD, Ross FM, Chiecchio L, Dagrada GP, Konn ZJ, Tapper WJ, et al. A novel prognostic model in myeloma based on co-segregating adverse FISH lesions and the ISS: analysis of patients treated in the MRC Myeloma IX trial. Leukemia. 2012;26:349-55.

11. Shah V, Sherborne AL, Walker BA, Johnson DC, Boyle EM, Ellis $\mathrm{S}$, et al. Prediction of outcome in newly diagnosed myeloma: a meta-analysis of the molecular profiles of 1905 trial patients. Leukemia. 2018;32:102-10.

12. Kumar SK, Rajkumar SV. The multiple myelomas - current concepts in cytogenetic classification and therapy. Nat Rev Clin Oncol. 2018;15:409-21.

13. An G, Xu Y, Shi L, Zou D, Deng S, Sui W, et al. t(11;14) multiple myeloma: a subtype associated with distinct immunological features, immunophenotypic characteristics but divergent outcome. Leuk Res. 2013;37:1251-7.

14. Chen L, Fan F, Deng J, Xu J, Xu A, Sun C, et al. Clinical characteristics and prognosis of immunoglobulin $\mathrm{D}$ myeloma in the novel agent era. Ann Hematol. 2019;98:963-70.

15. Swan D, Delaney C, Natoni A, O'Dwyer M, Krawczyk J. Successful venetoclax salvage in the setting of refractory, dialysisdependent multiple myeloma with $\mathrm{t}(11 ; 14)$. Haematologica. 2019; 105:e141-e143.

16. Smith D, Yong K. Advances in understanding prognosis in myeloma. Br J Haematol. 2016;175:367-80.

17. Goyal G, Rajkumar SV, Lacy MQ, Gertz MA, Buadi FK, Dispenzieri A, et al. Impact of prior diagnosis of monoclonal gammopathy on outcomes in newly diagnosed multiple myeloma. Leukemia. 2019;33:1273-7.

18. Fibbe WE, Jansen J. Prognostic factors in $\operatorname{IgD}$ myeloma: a study of 21 cases. Scand J Haematol. 1984;33:471-5.

19. Kim MK, Suh C, Lee DH, Min CK, Kim SJ, Kim K, et al. Immunoglobulin D multiple myeloma: response to therapy, survival, and prognostic factors in 75 patients. Ann Oncol. 2011; 22:411-6. 